\title{
Aptidão Física e Fatores de Risco Cardiometabólicos em Adolescentes: Um Estudo Longitudinal
}

\author{
Paula Roldão da Silva ${ }^{1}$, Waynne Ferreira de Faria ${ }^{2}$, Jadson Marcio da Silva ${ }^{3}$, \\ Géssika Castilho dos Santos ${ }^{4}$, Raphael Gonçalves de Oliveira' ${ }^{5}$, Antonio Stabelini Neto ${ }^{6}$
}

\begin{abstract}
RESUMO
Os níveis de aptidão física dos adolescentes são um importante indicador de seu estilo de vida e podem prevenir o desenvolvimento de fatores de risco cardiometabólicos. Este estudo teve como objetivo comparar os fatores de risco cardiometabólicos em adolescentes a partir das mudanças nos componentes de aptidão física relacionadas à saúde após três anos de seguimento. Participaram da pesquisa 93 adolescentes. Foram realizados testes motores segundo o manual do Fitnessgram (back-saver sit and reach; curl-up; push-up; e progressive aerobic cardiovascular endurance run), e avaliados os fatores de risco cardiometabólicos: circunferência de cintura, pressão arterial em repouso, HDL colesterol, triglicerídeos e glicemia em jejum em dois momentos (2014 e 2017). Para comparar os fatores de risco cardiometabólicos, desde as mudanças dos componentes de aptidão física entre os períodos de acompanhamento, foi aplicado o teste de Kruskal-Wallis ou Anova one-way. Foi observada melhora significativa dos componentes de AFRS com o passar dos anos, com exceção da aptidão cardiorrespiratória. Não foram identificadas diferenças estatísticas nos fatores de risco cardiometabólicos a partir da manutenção ou mudanças dos componentes de aptidão física relacionadas à saúde durante o período de acompanhamento.
\end{abstract}

Palavras-chave: Aptidão física. Aptidão cardiorrespiratória. Composição corporal. Saúde.

PHYSICAL FITNESS AND CARDIOMETABOLIC RISK FACTORS IN ADOLESCENTS: A LONGITUDINAL STUDYS

\section{ABSTRACT}

The physical fitness levels of adolescents are an important indicator of their lifestyle and can prevent the development of cardiomethabolic risk factors. The present study has the intention to compare the cardiomethabolic risk factors in adolescents from the changes in the physical fitness components related to health after three years of tracing. Participated in the research 93 adolescents. Motor tests were applied according to the Fitnessgram manual (back-saver sit and reach; curl-up; push-up; and progressive aerobic cardiovascular endurance run) and the cardiomethabolic risk factors were evaluated: waist circumference; resting blood pressure; colesterol HDL; triglycerides, and blood sugar in fast in two moments (2014 and 2017). In order to compare the cardiomethabolic risk factors from the changes in the physical fitness components during the tracing period the test Kruskal-Wallis or Anova one-way was applied. A significant improvement of the AFRS components was observed through the years, except to the cardiorespiratory fitness. No differences in the cardiomethabolic risk factors were identified from the maintenance of the physical fitness components related to health during the tracing period.

Keywords: Physical fitness. Cardiorespiratory fitness. Body composition. Health.

RECEBIDO EM: 20/9/2019

MODIFICAÇÕES SOLICITADAS EM: 8/11/2019

ACEITO EM: 23/11/2019

\footnotetext{
Mestrado em Educação Física pelo Programa de Pós-Graduação Associado UEM/UEL. http://lattes.cnpq.br/4098445248034531. https://orcid.org/00000002-7219-4946. proldaosilva@gmail.com

2 Doutorado em Educação Física pelo Programa de Pós-Graduação Associado UEM/UEL (2019). http://lattes.cnpq.br/5015504173344000. http://orcid. org/0000-0003-3407-6372. fariawf@outlook.com

${ }^{3}$ Bacharel em Educação Física. http://lattes.cnpq.br/5840838231212115. https://orcid.org/0000-0002-1955-4922. jadson_marcio@hotmail.com

${ }^{4}$ Doutoranda em Educação Física pelo Programa de Pós-Graduação em Associado em Educação Física - UEM/UEL pela Universidade Estadual de Londrina. http://lattes.cnpq.br/8267240903797006. https://orcid.org/0000-0003-1593-454. gessika.castilho@gmail.com

${ }^{5}$ Doutorado em Ciências da Reabilitação pela Universidade Norte do Paraná. http://lattes.cnpq.br/6247518975926252. https://orcid.org/0000-0003-09049305.rgoliveira@uenp.edu.br

${ }^{6}$ Doutorado em Educação Física. Professor-associado na Universidade Estadual do Norte do Paraná. http://lattes.cnpq.br/8550924080798366. https://orcid. org/0000-0003-2931-943X. asneto@uenp.edu.br
} 


\section{INTRODUÇÃO}

Informações da Pesquisa Nacional de Saúde do Brasil revelam que cerca de 57,4 milhões de pessoas, o equivalente a $40 \%$ da população adulta brasileira, possuem, pelo menos, uma doença crônica não transmissível, sendo estas doenças responsáveis por mais de $72 \%$ das causas de mortes no Brasil (IBGE, 2013). Essa condição é semelhante em outros países e tem aumentado substancialmente com o passar dos anos, e uma das prioridades dos órgãos de saúde é identificar comportamentos e tratamentos que possam reduzir os riscos a essas doenças, tanto a curto quanto em longo prazo, especialmente porque a detecção precoce pode ajudar a identificar os indivíduos com maior necessidade de intervenção (BUCHAN et al., 2015; SCHMIDT et al., 2016).

Estudos têm revelado que níveis adequados dos componentes da aptidão física relacionada à saúde (AFRS), ou seja, da aptidão cardiorrespiratória, composição corporal, flexibilidade, força e resistência muscular, estão associados à maior disposição para as atividades da vida diária, e atuam como fatores protetores contra as doenças crônicas não transmissíveis (AMERICAN..., 2011; BLAIR et al., 1995; BUCHAN et al., 2015).

Níveis adequados de aptidão física na infância e adolescência mostram-se inversamente relacionados aos mecanismos metabólicos que favorecem ou induzem o desenvolvimento destas disfunções (BLAIR et al., 1995; MATOS et al., 2016; ORTEGA et al., 2012). Ademais, pesquisadores sugerem que bons indicadores de AFRS durante a infância e adolescência podem prevenir o desenvolvimento de fatores de risco cardiometabólicos na idade adulta (ATTARD et al., 2013; FRASER et al., 2016; JUONALA et al., 2011; SCHMIDT et al., 2016). Logo, a literatura tem demonstrado a importância de se acompanhar os componentes de AFRS desta população, visando à sua relação com melhores condições de saúde.

É evidente, contudo, que os estudos realizados em adolescentes são predominantemente transversais, e dentre os estudos longitudinais realizados há uma grande variação no tempo de acompanhamento, o que dificulta a interpretação dos resultados, pois, em sua maioria, os benefícios são apresentados da infância ou adolescência à fase adulta (BUCHAN et al., 2015; JUHOLA et al., 2011; JUONALA et al., 2011; PÉREZ-BEY et al., 2018; PETKEVICIENE et al., 2015; SCHMIDT et al., 2016). Deste modo, notamos a necessidade de investigar o comportamento dos componentes de AFRS, em menor tempo de seguimento, em dife- rentes momentos da adolescência, pois este é considerado um período oportuno para o desenvolvimento dessas condições. O objetivo do presente trabalho, portanto, foi comparar os fatores de risco cardiometabólicos entre 2014 e 2017, a partir das mudanças nos componentes de AFRS entre os três anos de seguimento.

\section{MÉTODOS}

\section{Participantes}

Para a elaboração desta pesquisa foram utilizadas informações contidas no banco de dados do Projeto "Síndrome Metabólica e Aptidão Física na Adolescência: Fatores Associados". Desta forma, foram listados 432 adolescentes de ambos os sexos que participaram da primeira fase do estudo no ano de 2014. Destes, 383 adolescentes foram localizados por meio de uma busca a partir da listagem de todos os alunos matriculados nos respectivos colégios. Pela recusa em participar na segunda fase da pesquisa, não localização nos dias agendados ou pelos critérios de exclusão, a amostra final foi composta por 93 escolares de ambos os sexos ( 56 meninas e 37 meninos, média de 16,5 \pm anos de idade) com conjunto de dados completos.

Os critérios de exclusão da pesquisa foram: (a) qualquer problema de saúde que possa afetar sua participação no estudo; (b) gravidez; (c) utilização de algum tipo de medicamento que possa induzir a modificações das variáveis do estudo; (d) não comparecimento à escola no dia agendado para coleta dos dados. Os protocolos de intervenção foram aprovados pelo Comitê de Ética em Pesquisa da Universidade Norte do Paraná - Unopar (Parecer 1.302.963) e acompanharam normas da Resolução 466/12 do Conselho Nacional de Saúde sobre pesquisa envolvendo seres humanos.

\section{Procedimentos}

Tratando-se de um estudo longitudinal, a pesquisa atual consistiu da reavaliação de informações contidas do projeto anterior, portanto foram reaplicadas as mesmas avaliações realizadas no ano de 2014.

O protocolo de intervenção foi realizado na seguinte sequência: inicialmente, em jejum, foi realizada a coleta sanguínea para dosagens de lipídeos plasmáticos (HDL-C e triglicerídeos) e glicemia. Em seguida alimentaram-se e, após aproximadamente 15 minutos de repouso, foi aferida a pressão arterial. Na sequência, iniciaram a avaliação das medidas antropométricas (peso, estatura e circunferência de cintura) e, por 
fim, iniciaram a aplicação de testes motores para avaliação dos componentes de AFRS segundo o manual do Fitnessgram (MEREDITH; WELK, 2013) (back-saver sit and reach; curl-up; push-up; e progressive aerobic cardiovascular endurance run).

1. Back-saver sit and reach: o teste teve como objetivo avaliar a flexibilidade mediante a flexão do tronco à frente com uma perna estendida e a outra fletida.

2. Curl-up: avaliar a força e a resistência dos músculos da região abdominal por meio do movimento de flexão do tronco. O movimento foi feito mantendo-se o ritmo de uma repetição a cada três segundos, até a exaustão ou até o limite de 75 repetições.

3. Push-up: avaliar a força/resistência dos músculos dos membros superiores por intermédio do movimento de flexão e extensão dos cotovelos. O movimento foi feito mantendo-se o ritmo de uma repetição a cada três segundos, até a exaustão ou até o limite de 75 repetições.

4. Progressive aerobic cardiovascular endurance run: avaliar a aptidão cardiorrespiratória mediante corrida com mudanças de direção em um ritmo de intensidade progressivo. Cada estágio do teste possui duração de 1 minuto e o sinal sonoro (beep) é emitido progressivamente mais rápido a cada estágio, iniciando por volta de $8,5 \mathrm{~km} / \mathrm{h}$ no primeiro estágio e aumentando $0,5 \mathrm{~km} / \mathrm{h}$ por estágio. 0 teste foi encerrado quando os adolescentes interromperam voluntariamente seu deslocamento por exaustão, ou por não conseguirem manter o ritmo proposto pelo teste, sendo anotado o número de voltas completas. A estimativa do $\mathrm{VO}_{2 \text { máx }}$ foi realizada pela fórmula proposta por Saint-Maurice et al. (2015).

Esta sequência foi estipulada a fim de que a etapa anterior não influenciasse nos resultados da etapa subsequente. A coleta dos dados da atual pesquisa foi realizada de agosto a novembro de 2017, não havendo acompanhamento dos mesmos durante este período. As avaliações ocorreram sempre no período matutino, dentro do ambiente escolar.

\section{Análise estatística}

Os dados foram analisados no software estatístico SPSS for Windows versão 20.0, com um nível de significância estipulado em $p<0,05$. A normalidade dos dados foi avaliada pelo teste de Kolmogorov Smirnov. Para avaliar as diferenças das variáveis entre 2014 e 2017, foi utilizado o teste t para amostras dependentes ou Wilcoxon, e os valores foram apresentados de acordo com a normalidade (média e desvio padrão ou mediana e intervalo interquartis).
Para avaliar as alterações da aptidão física ao longo do estudo, foram criadas categorias de mudança a partir das classificações dos pontos de corte do Fitnessgram. As medidas foram agrupadas para, posteriormente, serem classificadas da seguinte forma: os classificados segundo o manual Fitnessgram em "zona saudável" foram agrupados como "alta aptidão", já os "precisa melhorar" e "precisa melhorar zona de risco" foram agrupados como "baixa aptidão". Os participantes que apresentavam baixa aptidão no início do estudo e permaneceram com baixa aptidão no segundo momento, foram classificados como "persistente baixo", aqueles que mudaram de alta aptidão para baixa foram classificados como "decrescente", aqueles que mudaram de baixa aptidão para alta foram classificados como "crescente" e aqueles que estavam com alta aptidão em ambos os momentos foram classificados como "persistente alto".

Com relação ao IMC, as medidas foram agrupadas para, posteriormente, serem classificadas da seguinte forma: os que foram selecionados segundo o manual Fitnessgram em "muito magro" e "zona saudável" foram agrupados como "peso normal", já os "precisa melhorar" e "precisa melhorar zona de risco" foram agrupados como "acima do peso" (classificação que também inclui obesidade). Desta forma, as alterações no IMC foram: os que permaneceram acima do peso em ambos os momentos foram classificados como "persistente acima", aqueles que estavam com peso normal e mudaram para acima do peso foram classificados como "crescente", os que estavam acima e mudaram para peso normal foram classificados como "decrescente" e os que mantiveram peso normal foram classificados como "persistente normal". Esta classificação foi utilizada para avaliar os fatores de risco cardiometabólicos de acordo com as alterações da aptidão física nos dois momentos, por meio do teste Kruskal-Wallis ou Anova One-way. Quando o teste $\mathrm{F}$ identificou significância estatística, o post hoc de Tukey foi aplicado para localizar as diferenças entre as médias.

\section{RESULTADOS}

As características dos participantes do estudo são apresentadas na Tabela 1. Nas variáveis de AFRS houve diferença estatística em todos os componentes. Os resultados indicaram melhora na flexibilidade, força e resistência da musculatura abdominal e de membro superior, aumento do IMC e diminuição do $\mathrm{VO}_{2 \text { máx }}$ entre os dois momentos. Para os fatores de risco cardiometabólicos avaliados, houve aumento na circunferência de cintura, pressão arterial diastólica, HDL e diminuição nas concentrações de triglicerídeos. 
Tabela 1 - Características dos participantes do estudo $(n=93)$

\begin{tabular}{|c|c|c|c|c|}
\hline \multirow[b]{2}{*}{ Variáveis } & 2014 & 2017 & \multirow[t]{2}{*}{$\Delta \%$} & \multirow[t]{2}{*}{ Valor de $\mathrm{P}$} \\
\hline & & & & \\
\hline Idade (anos) ${ }^{a}$ & $13,5 \pm 0,8$ & $16,5 \pm 0,8$ & 22,3 & $<0,001$ \\
\hline Peso $(\mathrm{Kg})^{\mathrm{a}}$ & $53,6 \pm 12,5$ & $60,3 \pm 12,2$ & 11,6 & $<0,001$ \\
\hline Estatura $(m)^{a}$ & $1,61 \pm 0,08$ & $1,66 \pm 0,09$ & 0,7 & $<0,001$ \\
\hline \multicolumn{5}{|c|}{ Medidas de Aptidão Física } \\
\hline Flexibilidade $(\mathrm{cm})^{a}$ & $17,7 \pm 6,4$ & $19,6 \pm 7,5$ & 10,3 & $<0,05$ \\
\hline F/R abdômen $(\mathrm{rpm})^{\mathrm{b}}$ & $16,5(9-27,75)$ & $23,0(14-36,5)$ & 102,1 & $<0,001$ \\
\hline F/R superior $(\mathrm{rpm})^{\mathrm{b}}$ & $0(0-1)$ & $6(0-12)$ & 0,0 & $<0,001$ \\
\hline $\mathrm{VO}_{2 \text { máx }}\left(\mathrm{ml} \cdot \mathrm{kg}^{-1} \cdot \mathrm{min}^{-1}\right)^{\mathrm{b}}$ & $37,6(35-41,17)$ & $34,9(31,80-39,93)$ & $-7,5$ & $<0,001$ \\
\hline $\mathrm{IMC}^{\mathrm{b}}$ & $19,5(18,03-23,14)$ & $21,0(19,52-24,24)$ & 7,8 & $<0,001$ \\
\hline \multicolumn{5}{|c|}{ Fatores de Risco Cardiometabólicos } \\
\hline $\mathrm{CC}(\mathrm{cm})^{\mathrm{a}}$ & $67,8 \pm 10,4$ & $71,9 \pm 9,22$ & 7,1 & $<0,001$ \\
\hline PAS $(\mathrm{mmHg})^{\mathrm{b}}$ & $100(100-110)$ & $100(100-110)$ & 0,0 & 0,364 \\
\hline PAD $(\mathrm{mmHg})^{\mathrm{b}}$ & $65(60-70)$ & $80(70-80)$ & 16,7 & $<0,001$ \\
\hline $\mathrm{TG}(\mathrm{mg} / \mathrm{dL})^{\mathrm{b}}$ & $81,7(76,60-85,70)$ & $66,4(51,45-93,65)$ & $-14,1$ & $<0,05$ \\
\hline $\mathrm{HDL}-\mathrm{C}(\mathrm{mg} / \mathrm{dL})^{\mathrm{a}}$ & $52,8 \pm 11,5$ & $56,4 \pm 10,7$ & 9,6 & $<0,001$ \\
\hline Glicemia $(\mathrm{mg} / \mathrm{dL})^{\mathrm{b}}$ & $80,5(77,15-85,10)$ & $80,8(78,30-85,40)$ & 0,6 & 0,978 \\
\hline
\end{tabular}

Nota: a dados paramétricos; ${ }^{b}$ dados não paramétricos; Abreviações: $\mathrm{F} / \mathrm{R}$ : força e resistência; $\mathrm{VO}_{2 \text { má: }}$ consumo máximo de oxigênio; $\mathrm{ml}$. $\mathrm{kg}^{-1}$. min ${ }^{-1}$ : mililitros por quilograma de peso por minuto; IMC: índice de massa corporal; CC: circunferência de cintura; PAS: pressão arterial sistólica; PAD: pressão arterial diastólica; TG: triglicerídeos; HDL: lipoproteína de alta densidade; cm: centímetros; rpm: repetições por minuto; mmHg: milímetros de mercúrio; mg/dL: miligramas por decilitro.

A Tabela 2 apresenta os resultados dos fatores de risco cardiometabólicos a partir das categorias de mudança do componente flexibilidade entre os anos de 2014 e 2017. Não foram observadas diferenças significativas entre os grupos.
Os resultados da Tabela 3 descrevem os fatores de risco cardiometabólicos a partir das categorias de mudança dos componentes força/resistência de abdômen e de membro superior entre os anos de 2014 e 2017. Não foram observadas diferenças significativas entre os fatores de risco cardiometabólicos pelos grupos de mudança com o passar dos anos.

Tabela 2 - Comparação dos fatores de risco cardiometabólicos entre as categorias de mudança para os níveis de flexibilidade

\begin{tabular}{|c|c|c|c|c|c|c|}
\hline \multicolumn{7}{|c|}{ Flexibilidade } \\
\hline Variável & $\begin{array}{c}\text { Persistente baixo } \\
\text { (65) }\end{array}$ & Decrescente (7) & Crescente (9) & $\begin{array}{c}\text { Persistente alto } \\
\text { (12) }\end{array}$ & $\mathbf{F}$ & $\mathbf{P}$ \\
\hline $\begin{array}{l}\text { Glicemia } \\
\text { (mg/dL) }\end{array}$ & $81,03 \pm 5,37$ & $86,16 \pm 5,90$ & $82,27 \pm 7,82$ & $82,86 \pm 4,23$ & 2,02 & 0,11 \\
\hline $\begin{array}{l}\text { TG } \\
(\mathrm{mg} / \mathrm{dL})\end{array}$ & $80,82 \pm 43,18$ & $74,74 \pm 35,92$ & $70,40 \pm 19,12$ & $67,48 \pm 33,12$ & 0,50 & 0,67 \\
\hline $\begin{array}{l}\text { HDL-C } \\
(\mathrm{mg} / \mathrm{dL})\end{array}$ & $54,65 \pm 10,25$ & $64,87 \pm 8,80$ & $56,40 \pm 13,59$ & $56,78 \pm 12,99$ & 1,90 & 0,13 \\
\hline $\begin{array}{l}\text { PAS } \\
(\mathrm{mmHg})\end{array}$ & $103,54 \pm 11,7$ & $102,86 \pm 7,55$ & $98,89 \pm 7,81$ & $109,17 \pm 9,96$ & 1,57 & 0,20 \\
\hline $\begin{array}{l}\text { PAD } \\
(\mathrm{mmHg})\end{array}$ & $76,46 \pm 8,55$ & $77,14 \pm 7,55$ & $75,56 \pm 10,13$ & $82,50 \pm 6,21$ & 1,87 & 0,13 \\
\hline $\begin{array}{l}\mathrm{CC} \\
(\mathrm{cm})\end{array}$ & $72,13 \pm 9,13$ & $70,74 \pm 8,24$ & $68,70 \pm 11,03$ & $74,34 \pm 9,24$ & 0,68 & 0,56 \\
\hline
\end{tabular}


Tabela 3 - Comparação dos fatores de risco cardiometabólicos entre as categorias de mudança entre força e resistência de abdômen e de membro superior

\begin{tabular}{|c|c|c|c|c|c|c|}
\hline \multicolumn{7}{|c|}{ Força e resistência de abdômen } \\
\hline Variável & $\begin{array}{l}\text { Persistente baixo } \\
\text { (29) }\end{array}$ & $\begin{array}{c}\text { Decrescente } \\
\text { (12) }\end{array}$ & Crescente (24) & $\begin{array}{l}\text { Persistente alto } \\
\text { (28) }\end{array}$ & $\mathbf{F}$ & $\mathbf{P}$ \\
\hline Glicemia (mg/dL) & $80,36 \pm 5,31$ & $81,43 \pm 4,63$ & $82,00 \pm 5,22$ & $83,19 \pm 6,55$ & 1,23 & 0,30 \\
\hline TG $(\mathrm{mg} / \mathrm{dL})$ & $87,88 \pm 50,95$ & $67,67 \pm 20,88$ & $66,24 \pm 28,90$ & $81,05 \pm 38,55$ & 1,66 & 0,18 \\
\hline $\mathrm{HDL}-\mathrm{C}(\mathrm{mg} / \mathrm{dL})$ & $52,71 \pm 11,52$ & $56,58 \pm 13,49$ & $55,90 \pm 7,66$ & $58,78 \pm 11,54$ & 1,48 & 0,22 \\
\hline PAS (mmHg) & $103,10 \pm 10,7$ & $107,50 \pm 7,53$ & $104,58 \pm 13,1$ & $102,14 \pm 11,0$ & 0,72 & 0,54 \\
\hline PAD (mmHg) & $77,93 \pm 7,73$ & $78,33 \pm 7,17$ & $77,08 \pm 9,99$ & $76,07 \pm 8,75$ & 0,30 & 0,82 \\
\hline $\mathrm{CC}(\mathrm{cm})$ & $74,99 \pm 11,32$ & $69,43 \pm 7,16$ & $70,45 \pm 8,17$ & $71,23 \pm 7,94$ & 1,64 & 0,18 \\
\hline \multicolumn{7}{|c|}{ Força e resistência de membro superior } \\
\hline Variável & $\begin{array}{c}\text { Persistente baixo } \\
\text { (71) }\end{array}$ & $\begin{array}{c}\text { Decrescente } \\
\text { (1) }\end{array}$ & Crescente (17) & $\begin{array}{c}\text { Persistente alto } \\
(4)\end{array}$ & $\mathbf{F}$ & $\mathbf{P}$ \\
\hline Glicemia (mg/dL) & $81,75 \pm 5,62$ & 83,30 & $82,19 \pm 6,24$ & $79,93 \pm 4,76$ & 0,19 & 0,90 \\
\hline TG (mg/dL) & $79,92 \pm 40,55$ & 54,90 & $76,62 \pm 39,27$ & $46,95 \pm 4,39$ & 0,99 & 0,40 \\
\hline $\mathrm{HDL}-\mathrm{C}(\mathrm{mg} / \mathrm{dL})$ & $54,38 \pm 10,46$ & 63,60 & $60,91 \pm 12,22$ & $58,85 \pm 12,53$ & 1,93 & 0,13 \\
\hline PAS (mmHg) & $104,37 \pm 10,5$ & 110,0 & $102,35 \pm 14,3$ & $97,50 \pm 5,0$ & 0,68 & 0,56 \\
\hline PAD (mmHg) & $77,89 \pm 8,43$ & 80,0 & $75,29 \pm 8,74$ & $72,50 \pm 9,57$ & 0,87 & 0,45 \\
\hline $\mathrm{CC}(\mathrm{cm})$ & $73,08 \pm 9,73$ & 77,50 & $69,05 \pm 6,07$ & $63,75 \pm 5,66$ & 2,16 & 0,09 \\
\hline
\end{tabular}

Nota: TG: triglicerídeos; HDL: lipoproteína de alta densidade; PAS: pressão arterial sistólica; PAD: pressão arterial diastólica; CC: circunferência de cintura.

Tabela 4 - Comparação dos fatores de risco cardiometabólicos entre as categorias de mudança do $\mathrm{VO}_{2 \text { máx }}$ VO ${ }_{2 \text { máx }}$

\begin{tabular}{|c|c|c|c|c|c|c|}
\hline Variável & $\begin{array}{c}\text { Persistente baixo } \\
\text { (59) }\end{array}$ & $\begin{array}{c}\text { Decrescente } \\
\text { (20) }\end{array}$ & $\begin{array}{c}\text { Crescente } \\
\text { (3) }\end{array}$ & $\begin{array}{c}\text { Persistente alto } \\
\text { (11) }\end{array}$ & $F$ & $\mathbf{P}$ \\
\hline Glicemia (mg/dL) & $82,14 \pm 5,57$ & $81,45 \pm 6,69$ & $81,73 \pm 3,63$ & $80,39 \pm 4,67$ & 0,32 & 0,81 \\
\hline TG (mg/dL) & $83,81 \pm 46,21$ & $68,01 \pm 19,73$ & $52,33 \pm 14,29$ & $68,88 \pm 24,36$ & 1,48 & 0,22 \\
\hline HDL-C (mg/dL) & $55,57 \pm 11,99$ & $57,35 \pm 10,07$ & $58,50 \pm 5,74$ & $53,99 \pm 8,75$ & 0,28 & 0,83 \\
\hline PAS (mmHg) & $103,39 \pm 10,6$ & $104,0 \pm 13,13$ & $106,67 \pm 11,54$ & $104,55 \pm 11,28$ & 0,10 & 0,95 \\
\hline PAD (mmHg) & $77,46 \pm 9,02$ & $76,0 \pm 5,98$ & $73,33 \pm 5,77$ & $79,09 \pm 10,44$ & 0,52 & 0,66 \\
\hline $\mathrm{CC}(\mathrm{cm})$ & $73,71 \pm 10,13$ & $68,39 \pm 7,03$ & $70,83 \pm 4,04$ & $69,21 \pm 6,02$ & 2,06 & 0,11 \\
\hline
\end{tabular}

Nota: $\mathrm{VO}_{2 \text { max }}$ : consumo máximo de oxigênio; TG: triglicerídeos; HDL: lipoproteína de alta densidade; PAS: pressão arterial sistólica; PAD: pressão arterial diastólica; CC: circunferência de cintura.

Tabela 5 - Comparação dos fatores de risco cardiometabólicos entre as categorias de mudança do IMC

\begin{tabular}{|c|c|c|c|c|c|c|}
\hline \multicolumn{7}{|l|}{ IMC } \\
\hline Variável & $\begin{array}{c}\text { Persistente acima } \\
\text { (28) }\end{array}$ & Crescente (3) & $\begin{array}{c}\text { Decrescente } \\
(9)\end{array}$ & $\begin{array}{c}\text { Persistente normal } \\
\text { (53) }\end{array}$ & $F$ & $\mathbf{P}$ \\
\hline Glicemia (mg/dL) & $81,36 \pm 5,98$ & $86,86 \pm 5,99$ & $81,44 \pm 5,42$ & $81,75 \pm 5,49$ & 0,87 & 0,45 \\
\hline TG (mg/dL) & $\begin{array}{c}64,70 \\
(48,05-115,67)\end{array}$ & $\begin{array}{c}96,50 \\
(67,60)\end{array}$ & $\begin{array}{c}68,00 \\
(54,40-83,55)\end{array}$ & $\begin{array}{c}67,20 \\
(51,80-79,85)\end{array}$ & $¥$ & 0,49 \\
\hline HDL-C (mg/dL) & $53,22 \pm 11,11$ & $54,43 \pm 12,00$ & $54,65 \pm 7,32$ & $57,53 \pm 11,42$ & 0,99 & 0,40 \\
\hline PAS (mmHg) & $\begin{array}{c}106,78 \pm \\
10,90\end{array}$ & $\begin{array}{c}106,66 \pm \\
11,54\end{array}$ & $\begin{array}{c}101,11 \pm \\
11,66\end{array}$ & $\begin{array}{c}102,45 \pm \\
11,07\end{array}$ & 1,18 & 0,32 \\
\hline PAD (mmHg) & $77,14 \pm 8,96$ & $80,00 \pm 10,00$ & $74,44 \pm 8,81$ & $77,54 \pm 8,29$ & 0,44 & 0,72 \\
\hline $\mathrm{CC}(\mathrm{cm})$ & $\begin{array}{c}77,25 \\
(65,77-80,50)^{*}\end{array}$ & $\begin{array}{c}79,0 \\
(73,50) \\
\end{array}$ & $\begin{array}{c}75,50 \\
(71,5-78,70)^{*} \\
\end{array}$ & $\begin{array}{c}68,50 \\
(64,25-72,00) \\
\end{array}$ & $¥$ & 0,00 \\
\hline
\end{tabular}

Nota: TG: triglicerídeos; HDL: lipoproteína de alta densidade; PAS: pressão arterial sistólica; PAD: pressão arterial diastólica; CC: circunferência de cintura. $¥:$ teste Kruskal-Wallis; *: diferença significativa $(p<0,001)$ comparada ao grupo persistente normal. 
Os resultados da Tabela 4 descrevem os fatores de risco cardiometabólicos isolados a partir das categorias de mudança do componente $\mathrm{VO}_{2 \text { máx }}$ entre os anos de 2014 e 2017. Não foram observadas diferenças estatísticas entre os grupos de mudança de $\mathrm{VO}_{2 \text { máx }}$.

A comparação dos fatores de risco cardiometabólicos, a partir das categorias de mudança do IMC entre os anos de 2014 e 2017, é apresentada na Tabela 5 . Os valores de CC foram significativamente menores entre os indivíduos que permaneceram em classificação do IMC normal em ambos os momentos, comparados aos classificados como persistente acima e decrescente.

\section{DISCUSSÃO}

No presente estudo observamos melhora nos componentes de AFRS durante o período de acompanhamento (2014-2017), com exceção da aptidão cardiorrespiratória. Essa melhora nestes componentes é esperada por conta das alterações referentes ao ganho de massa muscular associado à maturação na adolescência (MALINA; BOUCHARD; BAR-OR, 2009).

Com relação aos fatores de risco cardiometabólicos, identificamos um aumento significativo na CC durante o seguimento, característico desta fase da vida em virtude do processo de crescimento físico. Quanto aos valores de perfil lipídico, embora o rastreamento dos níveis de lipídios e lipoproteínas seja recomendado, deve-se ter cautela nas interpretações, uma vez que estes marcadores sofrem influências de fatores hormonais, principalmente durante a puberdade (MALINA; BOUCHARD; BAR-OR, 2009), bem como associados às mudanças no estilo de vida dos adolescentes (MAGNUSSEN et al., 2011).

Nossos resultados não apontaram diferenças estatísticas nos fatores de risco cardiometabólicos quando os adolescentes foram estratificados por grupos de mudança dos componentes de AFRS com o passar dos anos, com exceção do IMC.

Com o objetivo de examinar se a aptidão cardiorrespiratória na infância atenua ou modifica os riscos cardiometabólicos em longo prazo, Schmidt et al. (2016) observaram que, independentemente do estado de obesidade infantil, os participantes que aumentaram sua ACR durante o seguimento tiveram uma prevalência substancialmente menor de síndrome metabólica, comparados aos que mantiveram baixa aptidão. Dwyer et al. (2009) indicaram que a proporção de indivíduos com fatores de risco cardiometabólicos foram maiores nos grupos que permaneceram com baixa aptidão física ou diminuíram ao longo do tempo, em comparação com os indivíduos que aumentaram ou permaneceram com alta aptidão após 19 anos de acompanhamento.

No mesmo sentido, outros autores têm identificado influência dos componentes de aptidão física nos fatores de risco isolados com o passar dos anos. Petkeviciene et al. (2015) observaram que o risco de síndrome metabólica, hiperglicemia e diabetes na fase adulta podem ser afetados pelo IMC na juventude, enquanto o risco de hipertensão arterial, triglicerídeos elevados e redução do HDL-C estão associados ao aumento do IMC com o passar dos anos.

Usando dados de quatro cortes longitudinais da infância até a idade adulta, Juonala et al. (2011) identificaram que o risco cardiometabólico das crianças com sobrepeso ou obesas que apresentavam peso normal na idade adulta, foram semelhantes àquelas que nunca foram obesas. Ademais, os indivíduos com maior adiposidade da infância até a idade adulta tinham um risco aumentado de DM tipo 2 (RR 5,4; IC $95 \%, 3,4$ a 8,5), HA (RR 2,7; IC 95\%, 2,2 a 3,3), níveis elevados de colesterol LDL (RR 1,8; IC 95\%, 1,4 a 2,3), níveis reduzidos de HDL-C (RR 2,1; IC 95\%, 1,8 a 2,5) e hipertrigliceridemia (RR 3,0; IC 95\%, 2,4 a 3,8), comparados àqueles com IMC normal quando crianças e adultos.

Os estudos supracitados, contudo, tiveram seguimento da infância à idade adulta, dificultando nossa comparação pelo diferente período de seguimento. Acreditamos que a oscilação dos níveis de aptidão no período de seguimento pode ter influenciado nossos resultados, uma vez que indivíduo classificado como persistente alto pode ter diminuído seu nível de aptidão em algum momento durante este período de três anos.

É evidente na literatura que a maioria dos estudos realizados em adolescentes são predominantemente transversais. Deste modo, ressaltamos a avaliação das mudanças dos componentes de AFRS nos fatores de risco cardiometabólicos ao longo do tempo como ponto forte do nosso trabalho. Há, no entanto, limitações que devem ser consideradas na interpretação destes achados. Por se tratar de um estudo longitudinal, tivemos uma grande perda amostral, pois muitos adolescentes não foram encontrados no seguimento ou não aceitaram participar da segunda etapa da pesquisa. Quanto aos testes aplicados para avaliação dos componentes de AFRS, sabemos que, por se tratar de testes indiretos, os resultados são influenciados pelo esforço do participante, podendo não ter advindo de total empenho dos mesmos, entretanto não consideramos esta questão uma limitação, pois a 
bateria de testes Fitnessgram é amplamente utilizada, com testes de fácil aplicação e interpretação, sendo descrito como método válido e confiável para avaliar a aptidão em crianças e adolescentes (MEREDITH; WELK, 2013; PLOWMAN; MEREDITH, 2013).

Com relação aos pontos de corte dos componentes da AFRS, a bateria de testes Fitnessgram (MEREDITH; WELK, 2013) usa padrões referenciados estabelecidos para representar um nível de aptidão que oferece um grau de proteção contra doenças hipocinéticas, considerando também o processo de maturação na interpretação dos resultados. O principal objetivo da bateria de testes, todavia, é avaliar o desempenho da aptidão dos alunos no ambiente escolar, assim é possível que haja uma falta de sensibilidade dos pontos de corte dos componentes da AFRS para desfechos cardiometabólicos, sendo necessários mais estudos com esse objetivo.

Torna-se importante ressaltar que não temos conhecimento de pesquisas anteriores que tenham investigado os fatores de risco cardiometabólicos isolados a partir das mudanças dos componentes de AFRS durante a adolescência. Desta forma, é necessário que mais pesquisas com diferentes períodos de seguimento sejam realizadas para a generalização dos resultados.

\section{CONCLUSÃO}

Em tese, não foram identificadas diferenças estatísticas nos fatores de risco cardiometabólicos a partir da manutenção ou mudanças dos componentes de AFRS durante o período de acompanhamento. Ademais, houve melhora significativa dos componentes de AFRS com o passar dos anos, com exceção da aptidão cardiorrespiratória.

\section{REFERÊNCIAS}

ATTARD, S. M. et al. Longitudinal trajectories of BMI and cardiovascular disease risk: the national longitudinal study of adolescent health. Obesity, v. 21, n. 11, p. 2.180-2.188, 2013.

AMERICAN COLLEGE OF SPORTS MEDICINE. Position Stand. Quantity and quality of exercise for developing and maintaining cardiorespiratory, musculoskeletal, and neuromotor fitness in apparently healthy adults: guidance for prescribring exercise. Medicine \& Science in Sports \& Exercise, v. 43, n. 7, p. 1.334-1.359, 2011.

BLAIR, S. N. et al. Changes in physical fitness and all-cause mortality. A prospective study of healthy and unhealthy men. Journal of the American Medical Association, v. 273, n. 14, 1995.

BUCHAN, D. S. et al. Relationships between cardiorespiratory and muscular fitness with cardiometabolic risk in adolescents. Research in Sports Medicine, v. 23, n. 3, p. 227-239, 2015.
DWYER, T. et al. Decline in Physical Fitness from childhood to adulthood associated with increased obesity and insulin resistance in adults. Diabetes Care, v. 32, n. 4, p. 683-687, 2009.

FRASER, B. J. et al. Childhood muscular fitness phenotypes and adult metabolic syndrome. Medicine \& Science in Sports \& Exercise, v. 48, n. 9, p. 1.715-1.722, 2016.

IBGE. Instituto Brasileiro de Geografia e Estatística. Pesquisa Nacional de Saúde 2013: percepção do estado de saúde, estilos de vida e doenças crônicas - Brasil, Grandes Regiões e Unidades da Federação. 2013. Disponível em: ftp://ftp. ibge.gov.br/PNS/2013/pns2013.pdf. Acesso em: 9 jul. 2017. JUHOLA, J. et al. Tracking of serum lipid levels, blood pressure, and body mass index from childhood to adulthood: the cardiovascular risk in young finns study. Journal of Pediatrics, v. 159, n. 4, p. 584-590, 2011.

JUONALA, M. et al. Childhood adiposity, adult adiposity, and cardiovascular risk factors. The New England Journal of Medicine, n. 365, p. 1.876-1.885, 2011.

MAGNUSSEN, C. G. et al. Factors affecting the stability of blood lipid and lipoprotein levels from youth to adulthood: evidence from the Childhood Determinants of Adult Health Study. Archives of Pediatrics and Adolescent Medicine, $\mathrm{n}$. 165, v. 1, p. 68-76, 2011.

MALINA, R. M.; BOUCHARD, C.; BAR-OR, O. Crescimento, maturação e atividade física. Tradução Samantha Stamatiu e Adriana Inácio Elisa. São Paulo: Phorte, 2009.

MATOS, L. S. et al. Gênese da aterosclerose em crianças e adolescentes: artigo de revisão. Connection Line, v. 1, n. 14, p. 27-34, 2016.

MEREDITH, M. D.; WELK, G. J. Fitnessgram/Activitygram: test administration manual. $4^{\text {th }}$ ed. Dallas, Texas: The Cooper Institute, 2013.

ORTEGA, F. B. et al. Muscular strength in male adolescents and premature death: cohort study of one million participants. British Medical Journal, v. 354, p. e7279, 2012.

PÉREZ-BEY, A. et al. The role of adiposity in the association between muscular fitness and cardiovascular disease. The Journal of Pediatrics, n. 18, p. 1-8, 2018.

PETKEVICIENE, J. et al. Anthropometric measurements in childhood and prediction of cardiovascular risk factors in adulthood: kaunas cardiovascular risk cohort study. BMC Public Health, v. 15, n. 218, p. 1-8, 2015.

PLOWMAN, S. A.; MEREDITH, M. D. FITNESSGRAM/ACTIVITYGRAM Reference Guide, Dallas: The Cooper Institute, v. 8, n. 1, $4^{\text {th }}$ ed., p. 8-55, 2013.

SAINT-MAURICE, P. F.; WELK, G. J.; FINN, K. J.; KAJ, M. Cross-Validation of a PACER Prediction Equation for Assessing Aerobic Capacity in Hungarian Youth. Research Quarterly for Exercise and Sport, v. 86, n. Suppl 1, p. S66-73, 2015.

SCHMIDT, M. D. et al. Childhood fitness reduces the longterm cardiometabolic risks associated with childhood obesity. International Journal of Obesity, v. 40, p. 1.134-1.140, 2016. 